\title{
MODAL ANALYSIS OF HYBRID SISAL/JUTE NATURAL FIBER POLYMER COMPOSITE BEAM
}

\author{
Madhu Vani. A ${ }^{1}$, Maheeja. $B^{2}$, Pratheep Reddy. $T^{3}$ \\ ${ }^{1}$ Student, Mechanical Engineering, S.V.C.E, Tirupati, Andhra Pradesh, India \\ ${ }^{2}$ Student, Mechanical Engineering, S.V.C.E, Tirupati, Andhra Pradesh, India \\ ${ }^{3}$ Assistant Professor, Mechanical Engineering, S.V.C.E, Tirupati, Andhra Pradesh, India
}

\begin{abstract}
In the last decades the composites have been widely used in the construction, automobile and aerospace industries. Among them, Natural fiber composites are emerging as low cost, lightweight and superior alternatives to synthetic fiber composites. This study related to the development of bio-degradable composites using Epoxy resin and Sisal/Jute fibers. The fibers are chemically treated at different percentages of $\mathrm{NaOH}$ i.e., $3 \%, 6 \%$ and $9 \%$. Tensile and flexural strength of the composites were determined using UTM. The natural frequencies of the cantilever composite beam were determined analytically using Euler's theory and numerically using Ansys 15.0. From the results, the results agree close to each other.
\end{abstract}

Keywords: Sisal Fiber, Jute Fiber, Alkali Treatment, Modal Analysis.

\section{INTRODUCTION}

Natural fiber composites are alternatives to synthetic fiber reinforced composites, since the former fibers possess many advantages, such as high specific strength, low cost, low density, biodegradability, renewability, good thermal and acoustic insulating properties. From the past decade jute, sisal, coir, kenaf, banana, hemp, palmyra, flax etc., natural fibers are used as reinforcement in polymer composites for various engineering applications.

For the past decade, enormous work has been carried out on the characterization of the mechanical and physical properties of natural fiber reinforced polymer matrix composites in the form of short fibers, random distribution and single reinforcement by varying the weight percentage of reinforcement and different chemical treatment of fibers. Sardar et.al. [1] Investigated the Tensile Test on Sisal Fiber Reinforced Polyster Composite Material, by varying the weight percentage of fiber i.e., 15, 25, 35 and $45 \%$ and results shows that improvement in tensile properties of $15 \%$, $25 \%, 35 \%$ and $45 \%$ by increasing the percentage of fiber. Karthikeyan et.al. [2] studied the impact behavior of coir reinforced epoxy composite treated with alkali. In this study, coir fibers treated with $\mathrm{NaOH}$ of $2,4,6,8$ and $10 \%$ for 10 days. For each group of fiber, coir length was 10, 20 and 30 $\mathrm{mm}$. from the study, it is observed that, alkali treated specimens showed an improvement in impact strength of $15 \%$ compared with untreated fiber. Rokbi et al. [3] have studied the flexural properties of Alfa fiber reinforced composites by the influence of alkalization, and observed alkali treated fibers exhibits better properties compared to the untreated fiber composite.

Siddika et al. [4] studied the mechanical behavior of Hybrid Jute-Coir Fiber Reinforced Composites at 5, 10, 15 and 20 wt $\%$ fiber loading. 1:1 ratio of fibers are utilized during composite manufacturing. Mechanical properties were evaluated using ASTM standards. The study presents that tensile strength and Young's modulus of the composites were decreased and increased respectively with an increase in fiber loading. Hardness, Flexural and impact properties were increased with an increase in fiber loading. 20\% fiber composite yielded the best set of mechanical properties compared to other composites.

Ravi et al, [5] studied Mechanical Characterization of Banana/Sisal Fiber Reinforced Hybrid Composites for Structural Application. In this study, the composites are prepared with untreated and akali treated fibers by varying the weight percentage of fibers. The study shows that tensile properties of the treated fiber were higher than those of untreated fiber reinforced composites. It is believed that the fiber treatment improved the interfacial interaction, thus resulting in good strength and stiffness of the biocomposites materials. Dixit et al. [6] studied the Mechanical Behaviour of Hybrid Coir/Sisal/Jute reinforced polyester composite, and observed that the properties of hybrid composites are better than unhybrid composites.

Structures will vibrate in the excessive oscillatory motion. because of interaction between the inertial and elastic properties of the materials within a structure is called resonance. Reducing vibration is a primary task in design process. The resonant amplitude of vibration is significantly influenced by damping which is more in fiber reinforced composite structures than metal structures due to the viscoelastic behavior.

From the literature survey, it is observed that the Jute and sisal fiber reinforced composites shows better mechanical properties by varying the weight percentages of fibers, and also the alkali treatment of fibers shows better results 
compared to the untreated fibers. Hybrid reinforced composites shows a great change in the properties of the composites. From the literature survey, it is observed that most of the work has been done on discontinuous and short form of fibers in discontinuous orientation. Very few work has been carried out in woven form of reinforcement, and also very few work has been carried out on vibrational characteristics of natural fiber reinforced composites.

\section{MATERIALS AND METHODS}

\section{1 Selection of Matrix And Reinforcement}

The Literature survey presents that very limited work has been done on Hybrid Sisal/Jute woven natural fiber composite. Epoxy resin LY 556 and Hardener HY951 were used in this study and procured from Zenith Industrial supplies, Bangalore. The Jute/Sisal fiber used in this study procured from Shiva exports, Tirunelveli, Tamilnadu.

\subsection{Method Of Preparation:}

Initially, the Jute/Sisal fibers is to be treated with different percentages i.e., $3 \%, 6 \%$ and $9 \%$ of $\mathrm{NaOH}$ solution with distilled water in a glass jar for 3 days, and then the treated fibers are to be washed with distilled water. Again, fibers were washed with dilute HCL to remove alkali particles and then with water. Finally these fibers were washed with distilled water until the fibers were alkali free. The alkali treated Sisal/jute fibers are weaved in the form of long strands. Hand layup technique is adopted in this study to prepare the composite.

First, for quick and easy removal of composite, mold release plastic sheet was put over the glass plate and wax was applied at the inner surface of the mold. Calculated amount of Epoxy resin and Hardener (ratio of 10:1 by weight) was thoroughly mixed in a glass beaker, and pour in a wooden mold. Then the woven fiber mat was distributed on the mixture. Then again resin was applied over the fiber mat to get the desired thickness. Another releasing plastic sheet is spread over and then pressed and pushed down with an iron roller to avoid and eliminate any air bubble. Precautions were taken to avoid formation of air bubbles.

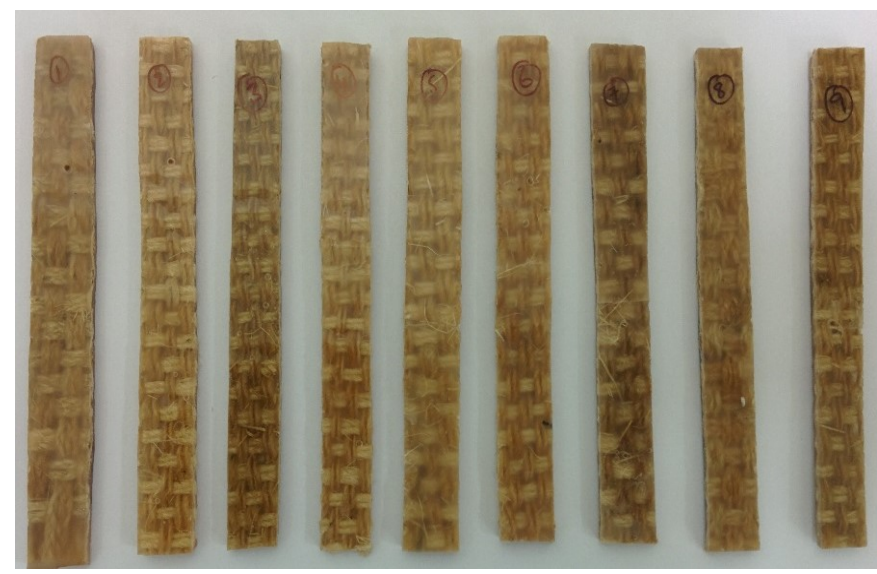

Fig-1: Sisal/Jute Hybid Composite

\subsection{Properties of the Composite}

The densities of the composite were investigated experimentally by the Archimedean's principle Tensile and flexural tests were carried out in an Instron (Series3382) testing machine according to ASTM: D3039-08 and ASTM D7264, respectively. An average of 3 specimens were used in each test.

\section{MODAL ANALYSIS:}

Modal analysis is one of the procedures determining the existent dynamic characteristic of a system in form of natural frequency, damping factors and mode shapes

\subsection{Analytical:}

The natural frequencies of the cantilever beam is calculated using Euler Bernoulli equation as given below.

$f_{n}=\frac{\beta_{n}^{2}}{2 n} \sqrt{\frac{E A}{\beta A I^{4}}}$

Where $\mathrm{fn}=$ natural frequency $(\mathrm{Hz}), \mathrm{E}=$ Youngs modulus (GPa), $\mathrm{A}=$ cross sectional Area $\left(\mathrm{m}^{2}\right) \rho=$ Density of the material $\left(\mathrm{kg} / \mathrm{m}^{3}\right), 1=$ length of the beam $(\mathrm{m}), \beta \mathrm{n}=$ constants for mode.

The dimensions of the cantilever beam used in this study is as follows: Length $=0.15 \mathrm{~m}$, Breadth $=0.01 \mathrm{~m}$, Depth $=$ $0.002 \mathrm{~m}$.

\subsection{Numerical Analysis:}

Finite element method is a numerical method which has solves the problems in various fields, like solid mechanics, fluid mechanics and heat transfer with approximate solutions. ANSYS 15.0 used in the present study for numerical study.

\section{Assumptions made for the present analysis were}

- $\quad$ Perfect bonding between fibers and matrix

- The composite lamina is free of voids and other irregularities

- The load is within the linear elastic limit

\subsubsection{Element Type}

The element SOLID186 used for the present study The element has 20 nodes having one degree of freedom i.e. temperature and with three degrees of freedom at each node: translation in the node $\mathrm{x}, \mathrm{y}$ and $\mathrm{z}$ directions respectively. 


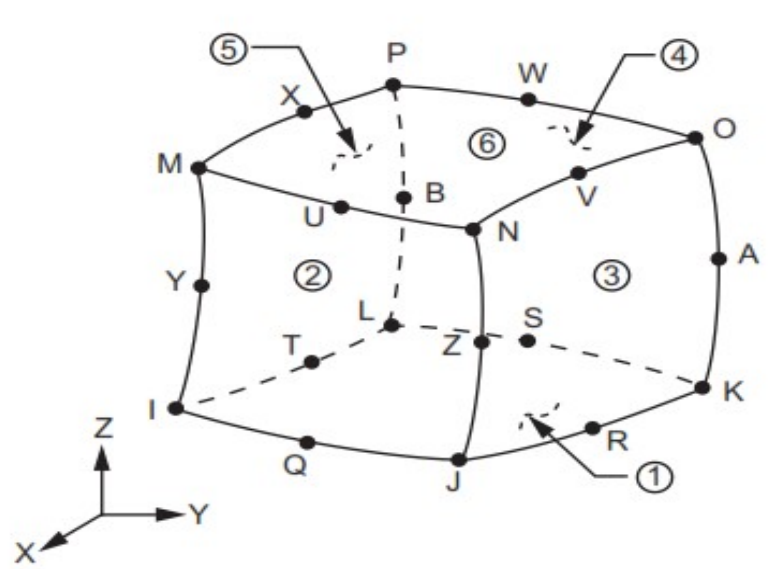

Fig -2: Element Solid 186

\subsubsection{Boundary Conditions}

For Cantilever Beam, the following symmetric boundary conditions are used. At $\mathrm{x}=0, \mathrm{Ux}=0$, At $\mathrm{y}=0, \mathrm{Uy}=0$, At $\mathrm{z}$ $=0, \mathrm{Uz}=0$.

\section{RESULTS AND DISCUSSION:}

Table -1: Density of the composites

\begin{tabular}{|l|l|l|}
\hline S. No & Specimen & Density $\left(\mathbf{k g} / \mathbf{m}^{\mathbf{3}}\right)$ \\
\hline & Untreated & 1300 \\
\hline & Resin + 3\% TF & 1290 \\
\hline & Resin $+6 \%$ TF & 1270 \\
\hline & Resin $+9 \%$ TF & 1240 \\
\hline
\end{tabular}

Table - 2: Tensile properties of the composites

\begin{tabular}{|l|l|l|l|}
\hline S. No & Specimen & $\begin{array}{l}\text { Tensile } \\
\text { strength } \\
\text { (Mpa) }\end{array}$ & $\begin{array}{l}\text { Tensile } \\
\text { Modulus } \\
\text { (Gpa) }\end{array}$ \\
\hline & Untreated & 82.5 & 0.54 \\
\hline & Resin $+3 \%$ TF & 86 & 1.23 \\
\hline & Resin $+6 \%$ TF & 89.5 & 1.89 \\
\hline & Resin $+9 \%$ TF & 97.5 & 2.62 \\
\hline
\end{tabular}

Table - 3: Flexural strength of the composites

\begin{tabular}{|l|l|l|}
\hline S. No & Specimen & Flexural strength (Mpa) \\
\hline & Untreated & 32 \\
\hline & Resin $+3 \%$ TF & 38 \\
\hline & Resin $+6 \%$ TF & 42 \\
\hline & Resin $+9 \%$ TF & 46 \\
\hline
\end{tabular}

From the above all results, it is observed that increase in percentage of alkali treatment improves the mechanical properties and natural frequencies of the composites, the reason for this is increase in alkali removes moisture from the fiber which improves the intermolecular hydrogen bonding between cellulose molecules in the fiber and water molecules, increases the interfacial adhesion between the fiber and the matrix.
Table - 4: Natural Frequencies of the composites

\begin{tabular}{|l|l|l|l|}
\hline $\begin{array}{l}\text { S. } \\
\text { No }\end{array}$ & Specimen & $\begin{array}{l}\text { Analytical } \\
(\mathbf{H z})\end{array}$ & $\begin{array}{l}\text { Numerical } \\
(\mathbf{H z})\end{array}$ \\
\hline & Untreated & 9.253 & 9.2891 \\
\hline & Resin + 3\% TF & 14.020 & 14.0737 \\
\hline & Resin +6\% TF & 17.515 & 17.5824 \\
\hline & Resin + 9\% TF & 20.870 & 20.9503 \\
\hline
\end{tabular}

From the above results, it is observed that the natural frequencies of the composites increased by increase in the percentage of alkali treatment of fiber. There is a close agreement between the analytical and numerical solitions.

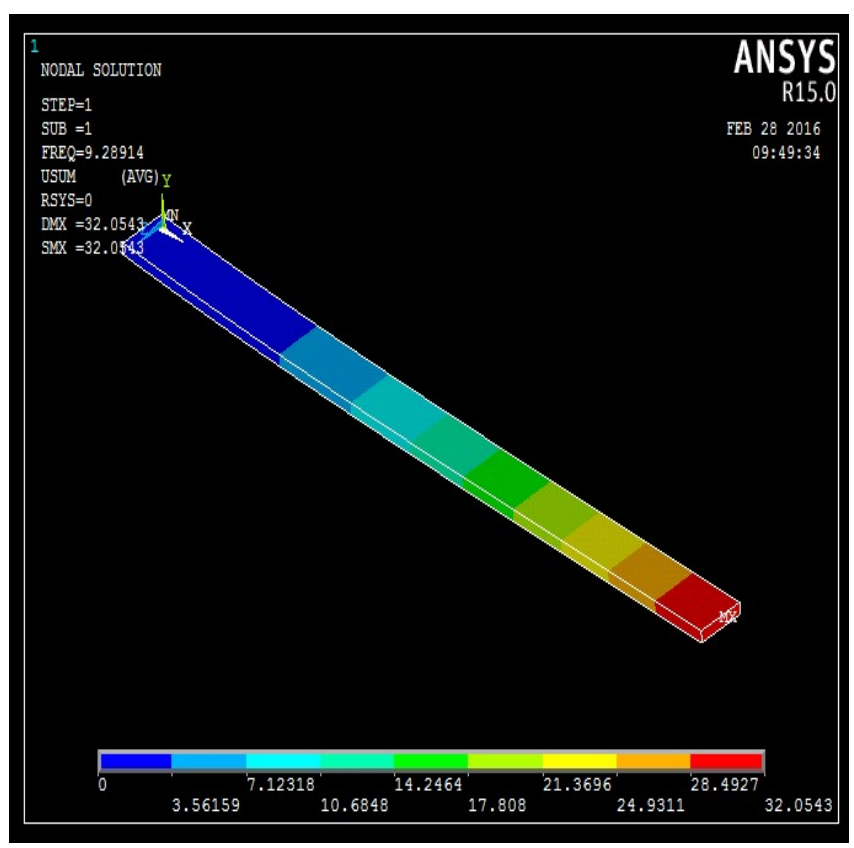

Fig -2: Natural Frequency of untreated fiber

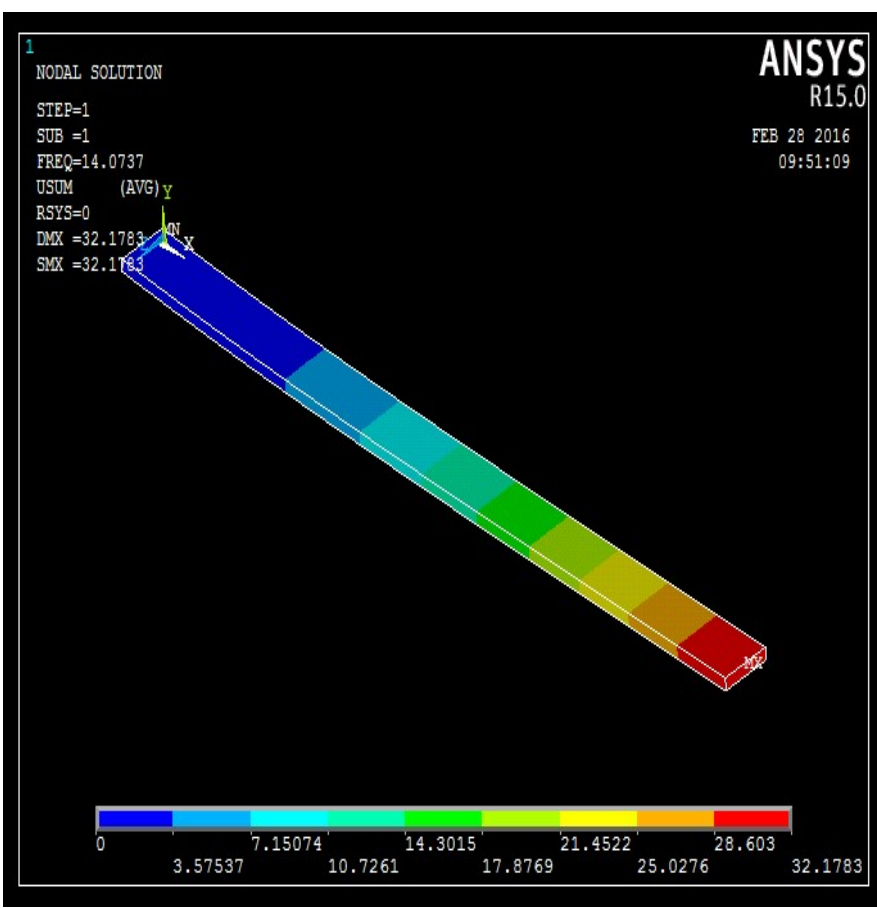

Fig - 3:Natural Frequency of 3\% alkali treated fiber 


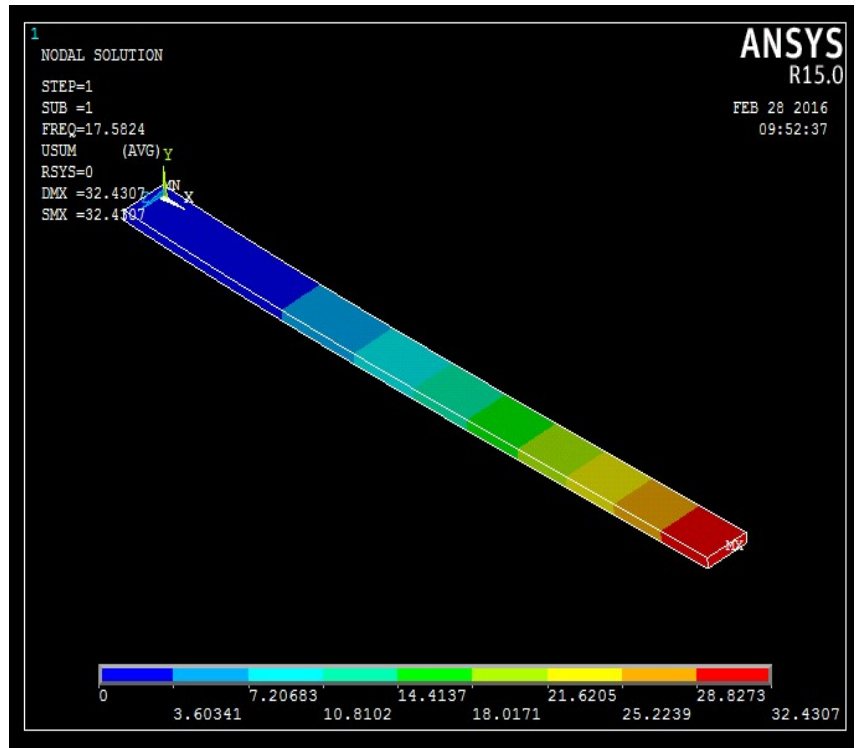

Fig -4: Natural Frequency of 6\% alkali treated fiber

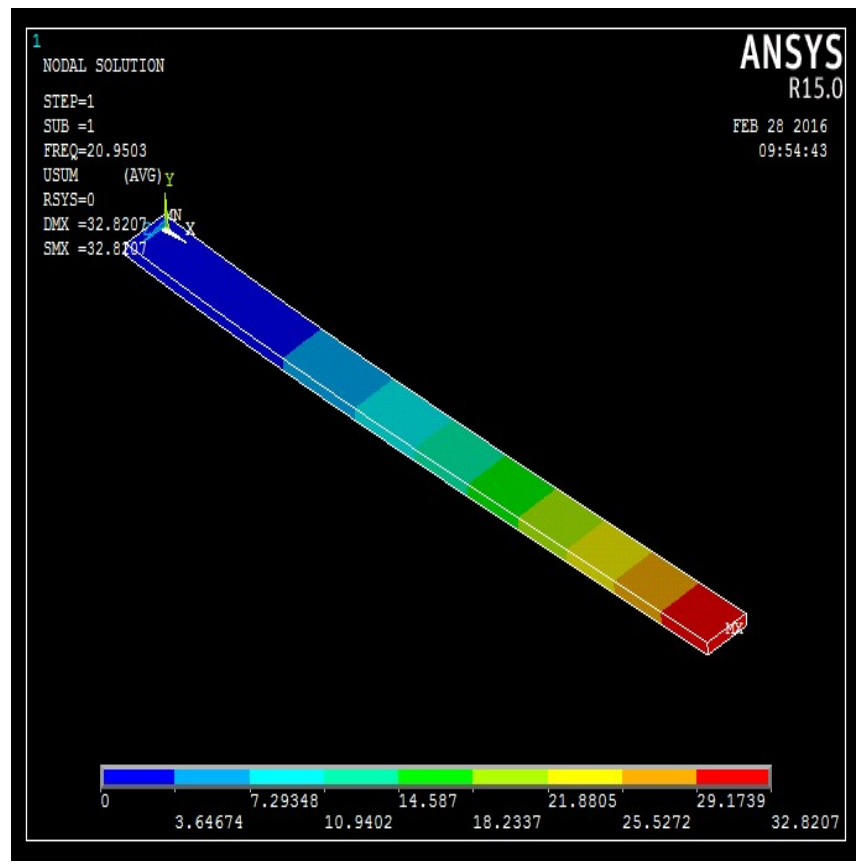

Fig -5: Natural Frequency of 9\% alkali treated fiber

\section{CONCLUSIONS}

- The alkali treated Sisal/jute natural fibers improved the properties of the fiber.

- Hybrid Composites were prepared successfully without any voids by hand layup process.

- From the study, the density of the composites decreases by increasing in the percentage of alkali treatment, the tensile strength and flexural strength of the composites increases by increasing in the percentage of alkali treatment.

- Bonding between the fiber and the resin improved by the removal of moisture.

- The natural frequency of the composites were determined using analytical and numerical approach. The results of the analytical and numerical agrees close to each other.

\section{REFERENCES:}

[1]. Kotresh sardar, K. Veeresh, T. Rangaswamy, Nandini $\mathrm{V}$ R, "Characterization and Investigation of Tensile Test on Sisal Fiber Reinforced Polyster Composite Material", International Journal of Recent Development in Engineering and Technology, 3(4), 2014, $84-89$.

[2]. Karthikeyan A, Bala Murugan K , "Effect of Alkali Treatment and fiber length on impact behavior of coir fiber reinforced epoxy composites, Journal f Scientific \& Industrial Research, 71, 2012, 627-631.

[3]. Mansour Rokbia, Hocine Osmania, Abdellatif Imadc , Noureddine Benseddiqd, "Effect of Chemical treatment on Flexure Properties of Natural Fiberreinforced Polyester Composite", Procedia Engineering 10 (2011) 2092-2097.

[4]. Salma Siddika, Fayeka Mansura, Mahbub Hasan "Physico-Mechanical Properties of Jute-Coir Fiber Reinforced Hybrid Polypropylene Composites" International Scholarly and Scientific Research \& Innovation 7(1), (2013), 60 -64.

[5]. Ravi Ranjan, P K Bajpai, \& R K Tyagi, "Mechanical Characterization of Banana/Sisal Fibre Reinforced PLA Hybrid Composites for Structural Application”, Engineering International, 1(1), 2013, 39 - 49.

[6]. Dixit S. Verma P. "The Effect of Hybridization on Mechanical Behaviour of Coir/Sisal/Jute Fibres Reinforced Polyester Composite Material", Research Journal of Chemical Sciences, 2(6), June 2012, 91-93.

[7]. A Textbook on Mechanical Vibrations, V.P.Singh, Dhanpat Rai \& Co.

[8]. ANSYS Mechanical APDL Element Reference. 